\title{
Modelo SIRC fracionário com múltiplas ordens para influenza
}

\author{
Fractional SIRC model with multi-term for influenza \\ A. C. F. N. Gomes*; A. De Cezaro \\ Instituto de Matemática, Estatística e Física, FURG, 96203-900, Rio Grande-RS, Brasil \\ *anagomes.mat@gmail.com
}

\begin{abstract}
Neste trabalho apresentaremos algumas variações do modelo compartimental SIRC proposto em [1] como uma alternativa interessante para a modelagem da evolução dinâmica da influenza. A primeira das principais contribuições está na escolha de uma dinâmica fracionária distinta para cada um dos compartimentos (S, I, R e C), respectivamente. Para tal modelo chamaremos de modelo SIRC fracionário. Tal dinâmica fracionária é bem conhecida por reproduzir efeitos de memória. Desta forma, estamos considerando efeito de memória (com distintos efeitos, haja vista que cada um dos componentes serão considerados com uma dinâmica fracionária distinta) no modelo SIRC. Provamos que o modelo SIRC fracionário é bem posto no sentido de Hadamard, i.e., possui uma única solução que depende continuamente do dados iniciais e dos parâmetros. A segunda das contribuições deste trabalho está em mostrar numericamente que, a partir de uma escolha adequada dos parâmetros no modelo SIRC fracionário proposto, a dinâmica reproduz de maneira mais condizente os dados reais de Influenza, coletados no estado do Rio Grande do Sul no ano de 2010 retirados do DATASUS. Conseguimos garantir que existe uma aproximação aos dados reais quando utilizamos derivadas de ordem fracionária segundo Caputo e mais, mostramos que a escolha de estudar o PVI fracionário com ordens fracionárias diferentes é a modelagem mais realista, pois quando consideramos ordens diferentes, a aproximação da curva $I(t)$ com os dados reais fica melhor.

Palavras-chave: Cálculo Fracionário, Modelo Compartimental SIRC, Memória Imunológica
\end{abstract}

In this paper we present some variations of the SIRC compartment model proposed in [1] as an interesting alternative for the modeling of the dynamic evolution of influenza. The first of the main contributions is in choosing a different fractional dynamics for each of the compartments (S, I, R and C), respectively. For this model we will call the Fractional SIRC model. Such fractional dynamics are well known for reproducing memory effects. In this way, we are considering memory effect (with different effects, since each of the components will be considered with a different fractional dynamics) in the SIRC model. We prove that the fractional SIRC model is well-positioned in the Hadamard sense, i.e., has a single solution that depends continuously on the initial data and the parameters. The second of the contributions of this work is to show numerically that from an appropriate choice of parameters in the proposed fractional SIRC model, the dynamics reproduces in a more appropriate way the actual Influenza data collected in the state of Rio Grande do Sul in the year of 2010 taken from DATASUS. We can guarantee that there is an approximation to the real data when we use fractional order derivatives according to Caputo. Moreover, we show that the choice of studying the fractional PVI with different fractional orders is the most realistic modeling, because when we consider different orders, the approximation of the curve $I(t)$ with the actual data gets better.

Keywords: Fractional Calculus, SIRC Compartmental Model, Immune Memory

\section{INTRODUÇÃO}

Existem várias definições de epidemiologia na literatura, oriundas de áreas diversas. Em resumo, epidemiologia é a ciência que estuda o processo de disseminação de doenças em certas populações, a fim de especificar fatores como: as causas de sua ocorrência, medidas de prevenção, controle e erradicação [2, 3].

Tendo em vista que epidemias são responsáveis, historicamente, por uma alta taxa de mortalidade em populações humanas, animais e vegetais, faz com que qualquer estudo no sentido de entender e dominar o progresso epidêmico seja justificável. Exemplos de tais epidemias são a Peste Negra, que assolou a Europa durante três anos e tem como estimativa de óbito de 30-50 \% da população infectada [4] ou a Cólera [5] que se espalhou pelo mundo em 1817. Desta forma, os estudos sobre epidemiologia tem se desprendido da área das ciências da saúde e adentrado nas pesquisas baseadas em modelos preditivos envolvendo conhecimentos estatísticos, computacionais e matemáticos [6, 7]. 
Usando desses artifícios e ainda respeitando as características biológicas dos sistemas, as pesquisas em sua maioria têm utilizado modelagens obtidas através de equações diferenciais [8], para melhor entender as interações entre os seres vivos e o meio ambiente. Estes procuram fornecer da forma mais fidedigna possível informações sobre os processos de disseminação e a dinâmica das doenças, assim como as formas de contágio e cura, buscando ser de grande auxílio na tomada de decisão dos órgãos governamentais. No presente trabalho, assumiremos que a dinâmica da influenza evolui de acordo com o modelo epidemiológico compartimental conhecido como SIRC. Em outras palavras, que a população total $N$ esteja dividida em 4 compartimentos: $S$ - de suscetíveis, $I$ - de infectados, $R$ - dos recuperados, e $C$ - dos indivíduos que possuem imunidade cruzada. O modelo SIRC foi proposto inicialmente por Casagrandi et al. (2006) [1] e é uma variação do já conhecido modelo SIR [9].

Basicamente, o modelo SIRC se diferencia do SIR por incluir o novo compartimento $C$, que representa os indivíduos que estão sujeitos a uma mutação da doença. Ou seja, leva em consideração os indivíduos que já foram infectados e que, após um tempo, recuperados e com imunidade passam a ser suscetíveis não a doença original, mas a uma mutação dela. Haja vista as mutações constantes do vírus da influenza faz jus à consideração do modelo SIRC como um modelo promissor para a modelagem.

Para uma boa visualização do comportamento desse modelo com este novo compartimento, apresentamos o Diagrama compartimental do modelo SIRC na Figura 1.

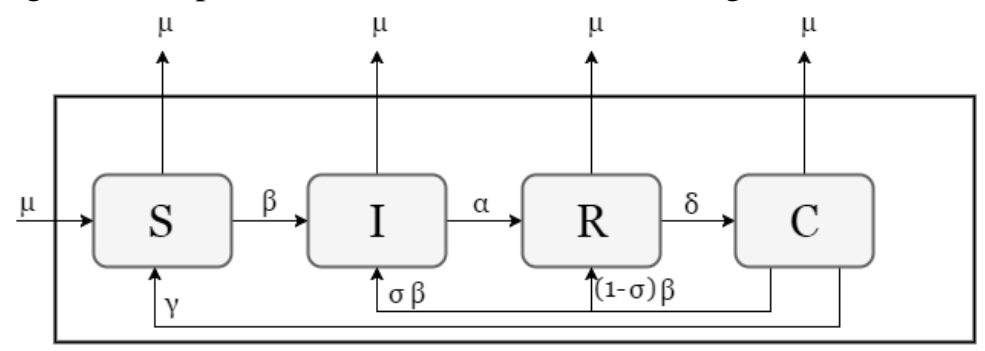

Figura 1: Diagrama Compartimental.

Uma de nossas contribuições está no estudo de modelos do tipo SIRC com efeitos de memória. Este efeito não é considerado no modelo SIRC proposto em Casagrandi et al. (2006) [1]. A particularidade da memória no sistema SIRC pode ser encarada da seguinte forma: o sistema imunológico tem sido conceitualmente dividido em imunidade inata e imunidade adaptativa [10]. A imunidade inata representa uma resposta rápida e estereotipada a um número grande, mas limitado, de estímulos e tem como principais células macrófagos, neutrófilos, células dendríticas e células Natural Killer [11]. Já a imunidade adaptativa, tem como principais células os Linfócitos $T$ e B, Natural Killer/T, Células dendríticas ou apresentadoras de antígeno [12]. Trata-se da defesa adquirida por contato com o invasor e é específica somente aquele invasor. Captar esse efeito, aqui chamado de memória, é uma das metas desse estudo.

Existem diversas maneiras de incorporar memória em sistemas dinâmicos como o modelo SIRC $[13,14]$. Nossa abordagem consiste em introduzir a memória através do uso de derivadas de ordem fracionária como dinâmica do modelo SIRC, ao qual chamaremos de modelo SIRC fracionário. É importante notar que o modelo SIRC fracionário já foi estudado em $[15,16,17]$. No entanto, o modelo SIRC cujas ordens das derivadas são distintas em cada linha do sistema ainda não foi explorado.

Como principais contribuições deste trabalho, vamos propor o modelo SIRC com derivadas de ordem fracionária distintas em cada linha do sistema, mudando a dinâmica que modela o problema. Em particular, tal abordagem permite propor distintos níveis de memória para cada um dos compartimentos do modelo SIRC. Provaremos resultados de boa colocação para o problema proposto e nas simulações mostraremos através da comparação numérica que o modelo SIRC fracionário proposto descreve, de maneira mais fidedigna, dados reais relativos à influenza.

Esta contribuição se divide da seguinte forma: primeiramente, na seção 2, apresentaremos o PVI fracionário Eq.(1) que aqui será estudado. Na seção 3, dividiremos os resultados, primeiramente mostraremos os resultados de boa colocação para o PVI fracionário Eq.(1) e posteriormente, 
traremos os resultados numéricos dessa proposta e suas discussões. Na seção 4 faremos uma breve conclusão do assunto e indicaremos os próximos passos dessa pesquisa.

\section{MATERIAL E MÉTODOS}

Neste trabalho estudaremos a generalização do modelo SIRC proposto por [1], em que a dinâmica é dada por operadores de ordem fracionária do tipo Caputo [13] $\left(D_{*}\right)$, que podem ser diferentes entre si, como pode ser observado no PVI fracionário Eq.(1):

$$
\begin{aligned}
& D_{*}^{\theta_{1}} S(t)=\mu(N-S(t))-\beta S(t) I(t)+\gamma C(t) \\
& D_{*}^{\theta_{2}} I(t)=\beta S(t) I(t)+\sigma \beta C(t) I(t)-(\mu+\alpha) I(t) \\
& D_{*}^{\theta_{3}} R(t)=(1-\sigma) \beta C(t) I(t)+\alpha I(t)-(\mu+\delta) R(t) \\
& D_{*}^{\theta_{4}} C(t)=\delta R(t)-\beta C(t) I(t)-(\mu+\gamma) C(t)
\end{aligned}
$$

No PVI fracionário Eq.(1), o parâmetro $\mu>0$ é a taxa de mortalidade e natalidade, $\alpha>0, \delta>$ $0, \gamma>0$ são o inverso do tempo em que os indivíduos ficam no compartimentos $I, R$ e $C$, respectivamente, $\sigma>0$ é a probabilidade média de reinfecção e , $\beta>0$ é o contato entre os compartimentos $S$ e $I$.

As quantidades $S(0)=S_{0}, I(0)=I_{0}, R(0)=R_{0}$ e $C(0)=C_{0}$ são as condições iniciais para o PVI fracionário Eq.(1). Já $N=S+I+R+C$, representando o total da população, e $\theta_{j} \in$ $[0,1]$ para $j=1, \ldots, 4$ são as ordens da derivada.

Em particular, quando $\theta_{j}=1$ o modelo é o proposto por [1] e para $\theta_{1}=\theta_{2}=\theta_{3}=\theta_{4}$ os resultados de boa colocação do PVI fracionário Eq.(1), com as condições iniciais acima podem ser encontrados em Gomes e De Cezaro (2017) [18].

\section{RESULTADOS E DISCUSSÃO}

Para colocarmos o PVI fracionário Eq.(1) de forma mais formal, temos:

$$
\begin{gathered}
\mathrm{D}_{*}^{\theta_{1}} \mathrm{u}_{1}(\mathrm{t})=\mathrm{f}_{1}\left(\mathrm{t}, \mathrm{u}_{1}(\mathrm{t}), \ldots, \mathrm{u}_{\mathrm{k}}(\mathrm{t})\right) \\
\vdots \\
\mathrm{D}_{*}^{\theta_{\mathrm{j}}} \mathrm{u}_{\mathrm{k}}(\mathrm{t})=\mathrm{f}_{1}\left(\mathrm{t}, \mathrm{u}_{1}(\mathrm{t}), \ldots, \mathrm{u}_{\mathrm{k}}(\mathrm{t})\right) \\
\mathrm{u}_{\mathrm{j}}(0)=\mathrm{u}_{\mathrm{j}, 0}(\mathrm{j}=1,2, \ldots, \mathrm{k})
\end{gathered}
$$

Para atingirmos nossos objetivos, basta assumirmos que $0<\theta_{j} \leq 1$ para todo $j$.

Teorema 1. Considere $\theta_{j}>0$ para $j=1,2, \ldots, k$ e considere o problema do valor inicial fracionário Eq.(1).

$$
\begin{aligned}
& D_{*}^{\theta_{j}}\left(u_{j}(t)\right)=f_{j}\left(t, u_{1}(t), \ldots, u_{k}(t)\right),(j=1,2, \ldots, k) \\
& u_{j}^{l}(0)=u_{j, 0}^{l}\left(l=0,1, \ldots,\left[\theta_{j}\right]-l\right)(j=1,2, \ldots, k)
\end{aligned}
$$

Suponha que as funções $f_{j}:[0, T] \times \mathbb{R}^{k} \rightarrow \mathbb{R}, j=1,2, \ldots, k$, sejam contínuas e satisfaçam as condições de Lipschitz em relação a todos os seus argumentos, exceto o primeiro. Então, o problema do valor inicial tem uma solução única que é continuamente dependente das condições iniciais, dos parâmetros e da ordem da derivada.

Demonstração 1. O problema de valor inicial Eq.(3), é equivalente ao sistema de equações de Volterra,

$$
u_{j}(t)=\sum_{l=0}^{\left[\theta_{j}\right]-1} u_{j, 0}^{l} \frac{t^{l}}{l !}+\frac{1}{\Gamma\left(\theta_{j}\right)} \int_{0}^{t}(t-s)^{\theta_{j}-1} f_{j}\left(s, u_{1}(s), \ldots, u_{k}(s)\right) d s,
$$

Em seguida, definimos $\theta=\min _{j} \theta_{j}$ e reescrevemos na equação anterior na forma:

$$
u_{j}(t)=\sum_{l=0}^{\left[\theta_{j}\right]-1} u_{j, 0}^{l} \frac{t^{l}}{l !}+\frac{1}{\Gamma(\theta)} \int_{0}^{t}(t-s)^{\theta_{j}-1} \hat{f}_{j}\left(s, u_{1}(s), \ldots, u_{k}(s)\right) d s,
$$


para $j=1,2, \ldots, k$, onde,

$$
\hat{f}_{j}\left(s, u_{1}, \ldots, u_{k}\right):=\frac{\Gamma(\theta)}{\Gamma\left(\theta_{j}\right)}(t-s)^{\theta_{j}-\theta} f_{j}\left(s, u_{1}, \ldots, u_{k}\right)
$$

Apresentando a notação vetorial $U:=\left(u_{1}, \ldots, u_{k}\right)^{T}, \hat{F}:=\left(\hat{f}_{1}, \ldots, \widehat{f}_{k}\right)^{T}$ e uma expressão correspondente para os valores iniciais, podemos combinar essas $k$ equações escalares em uma equação vetorial, da forma

$$
U(t)=\sum_{l=0}^{\max _{j}\left[\theta_{j}\right]-1} U_{0}^{l} \frac{t^{l}}{l !}+\frac{1}{\Gamma(\theta)} \int_{0}^{t}(t-s)^{\theta-1} \hat{F}(s, U(s)) d s,(6)
$$

Como $f_{j}:[0, T] \times \mathbb{R}^{k} \rightarrow \mathbb{R}, j=1,2, \ldots, k$ e o fato de que $\theta_{j} \geq \theta$ para todos $j$, concluímos que todas as funções $\hat{f}_{j}$ são contínuas e satisfazem as condições de Lipschitz com respeito a $u_{1}, \ldots, u_{k}$. Assim, $\hat{F}_{j}$ é contínua e satisfaz uma condição de Lipschitz em relação a $U$. Logo, com uma argumentação análoga a feita por Gomes e De Cezaro (2017) [18] está garantida a existência e unicidade de uma solução continuamente dependente $U=\left(u_{1}, \ldots, u_{k}\right)^{T}$.

A prova da dependência contínua, das múltiplas ordens da derivada, do PVI fracionário Eq.(1), trata-se de uma consequência da Eq. (6).

Basta que se considere um problema próximo ao PVI Eq.(3) só que tendo com ordem da derivada $\tilde{\theta}_{j}$, onde $\tilde{\theta}_{j}>\theta_{j}$ e $\varepsilon_{j}:=\tilde{\theta}_{j}-\theta_{j}$, como no seguinte resultado [Teorema 6.21, p. 113][13] Quando fôssemos pegar o $\theta=\min \theta_{j}$ pegássemos $\theta=\min \left\{\theta_{j}, \tilde{\theta}_{j}\right\}$. Portanto a dependência contínua das múltiplas ordens da derivada do PVI Eq.(3) é garantida.

Como no Brasil existem panoramas preocupantes principalmente por ter elevadas taxas de mortalidade por doenças infecciosas em comparação aos padrões mundiais [19], como por exemplo, a alarmante e rápida propagação do vírus Zika, no nordeste, trazendo consequências para mulheres [20,21] e homens [22], principalmente a sua associação aos casos de microcefalia em recém nascidos. Além de outras doenças como Chagas [23], Dengue [24], Febre Amarela [25] e principalmente diversas formas de influenzas [24], estudos sobre estas doenças são justificáveis.

Em 2009, o surgimento da influenza pandêmica [25] representou um desafio mundial para a vigilância em saúde, exigindo conhecimento sobre a doença e os meios para seu controle, estabelecendo medidas preventivas efetivas e tratamentos adequados.

O PVI fracionário Eq.(1) pode ser calibrado para várias doenças, desde que se conheça o valor de seus parâmetros biológicos. Nesta proposta trabalharemos com dados da influenza, já que o sistema DATASUS, sistema de tecnologia da informação a serviço do sistema único de saúde, disponibiliza informações sobre como procedeu ao contágio no Brasil.

Utilizamos para este trabalho os dados do estado do Rio Grande do Sul no ano de 2010. Tomando por base os parâmetros estudados por Casagrandi et al. (2006) [1], mas fazendo alguns ajustes, tendo em vista a melhor representação dos dados.

Alteraremos o parâmetro responsável pelo tempo em que os indivíduos ficam infectados $\alpha$ e a taxa de contato entre os suscetíveis e infectados $\beta$, utilizando um quinto dos valores originais. Os parâmetros utilizados para as simulações são apresentados na Tabela 1.

Tabela 1: Parâmetros da literatura e dessa proposta

\begin{tabular}{ccc}
\hline \multirow{2}{*}{ Parâmetros } & \multicolumn{2}{c}{ Parâmetros da influenza } \\
\cline { 2 - 3 } & Casagrandi et al. [1] & Nossos valores \\
\hline $\boldsymbol{\mu}$ & 0,02 & 0,02 \\
$\boldsymbol{\alpha}$ & 52,15 & 10,43 \\
$\boldsymbol{\delta}$ & 0,75 & 0,75 \\
$\boldsymbol{\gamma}$ & 0,35 & 0,35 \\
$\boldsymbol{\sigma}$ & 0,12 & 0,12 \\
$\boldsymbol{\beta}$ & 1,35 & 0,27 \\
$\boldsymbol{N}$ & 100 & 100 \\
\hline
\end{tabular}


A Figura 2, expõe a dinâmica do PVI fracionário Eq.(1) em comparação com a curva dos dados reais, utilizando $\theta_{1}=\theta_{2}=\theta_{3}=\theta_{4}=1$, ou seja, como se o modelo Eq.(1) tivesse ordem inteira. Os dados reais nos mostram a quantidade de infectados ao longo do tempo, logo a curva que esperamos que se aproxime dos dados é a $I(t)$.

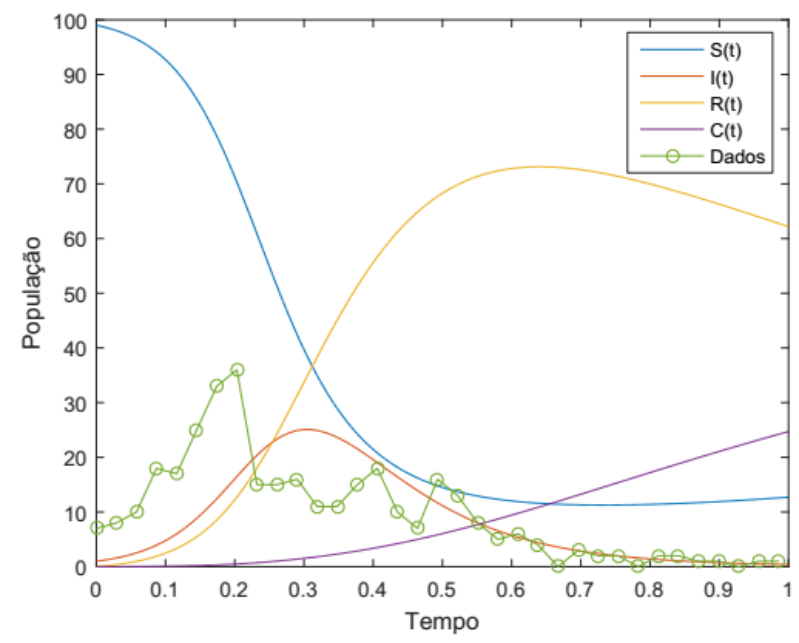

Figura 2: Ordem inteira.

Como fica visível a curva $I(t)$ não se aproxima de forma satisfatória dos dados reais. Além disso, este modelo não leva em conta o efeito da memória imunológica. A população fica rapidamente infectada e a curva $C(t)$, que representa os indivíduos que estão sujeitos a uma nova cepa da doença, tem um comportamento crescente.

Na Figura 3, apresentamos a dinâmica do PVI fracionário Eq.(1) em comparação com a curva dos dados reais, com $\theta_{1}=\theta_{2}=\theta_{3}=\theta_{4}=0$,8. Esse valor foi escolhido dentre tantas ordens fracionárias possíveis, por tentarmos captar o efeito de memória sem que seja demasiadamente forçado. Os dados reais são os mesmos utilizados na Figura 2, porém agora notamos uma aproximação da curva $I(t)$ com os dados reais.

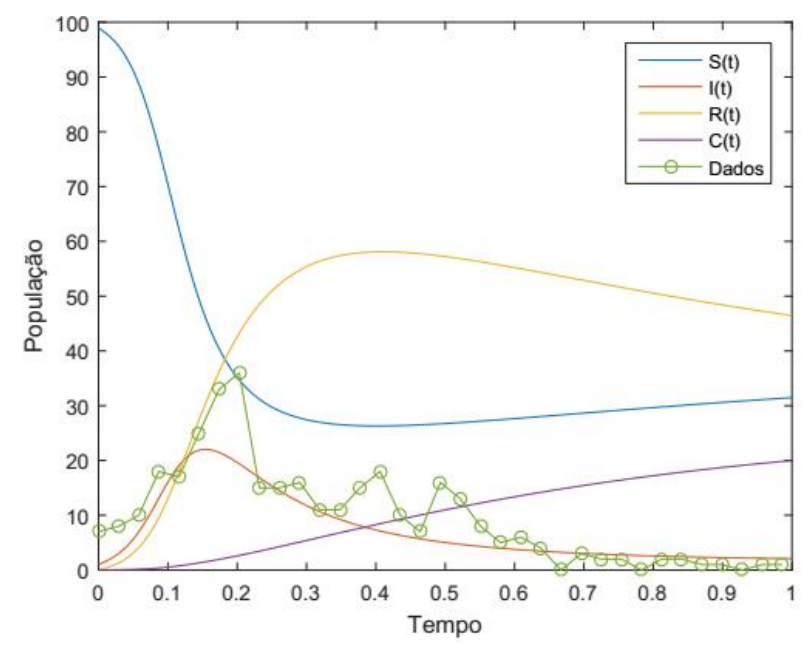

Figura 3: Ordem fracionária igual.

Observamos que com o uso de $\theta_{1}=\theta_{2}=\theta_{3}=\theta_{4}=0,8$, parece que existe um impedimento maior, até um pouco exagerado, para que a população se torne infectada. Acreditamos que o ideal é considerar uma diferenciação da ordem das derivadas entre as linhas do sistema, tendo como hipótese, e.g., que a população do compartimento imunidade cruzada possuí mais memória imunológica do que o compartimento dos suscetíveis.

A dinâmica do sistema representada na Figura 4 tem algumas hipóteses biológicas interessantes de serem discutidas quanto à escolha das ordens das derivadas. Para a primeira linha do PVI 
fracionário Eq.(1) escolhemos $\theta_{1}=0,99$ tendo em vista que é a linha que representa o compartimento dos suscetíveis. A ideia é utilizar uma pequena memória imunológica, já que os indivíduos estão sempre em contato com vários estímulos.

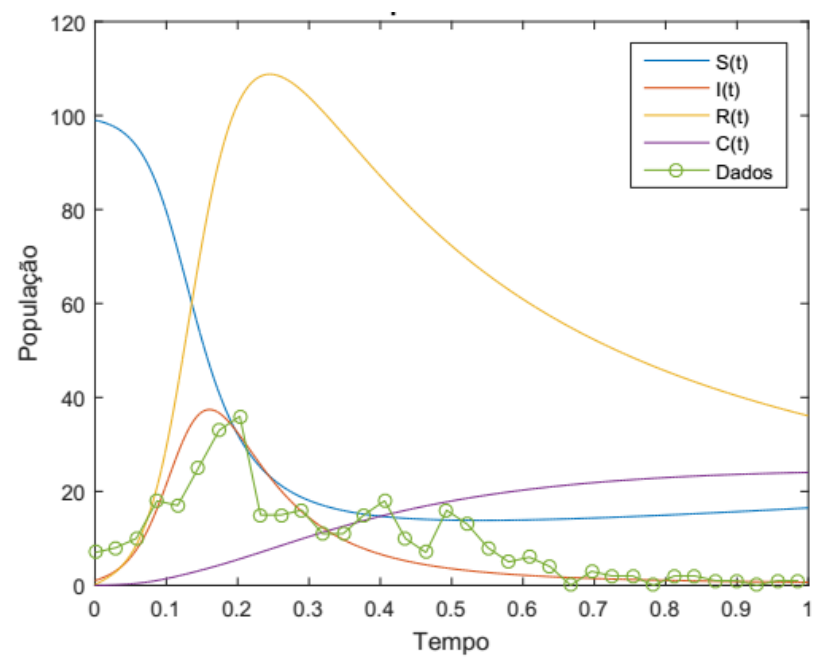

Figura 4: Ordem fracionária diferente.

Na segunda linha do PVI fracionário Eq.(1), foi utilizado $\theta_{2}=0,8$ já que é a linha que representa o compartimento dos infectados, onde a população tem contato com o patógeno e neste momento acreditamos que a memória imunológica cresce bastante.

A terceira linha do PVI fracionário Eq.(1) representa os indivíduos em recuperação e onde ficam imunes ao patógeno. Acreditados que a memória imunológica está mais desenvolvida nesta fase, logo a ordem da derivada utilizada foi $\theta_{3}=0,6$.

Na última linha do PVI fracionário Eq.(1), utilizamos $\theta_{4}=0,7$, pois nesse compartimento parte dos indivíduos perdem a imunidade, $\operatorname{logo}$ acreditamos que a memória imunológica diminui. Percebemos então que a Figura 4 apresenta a simulação que mais se aproxima dos dados reais dentre os casos estudados, então podemos afirmar que é uma boa aproximação da realidade.

Na Figura 5, estamos exibindo a comparação de cada um dos casos comentados anteriormente.

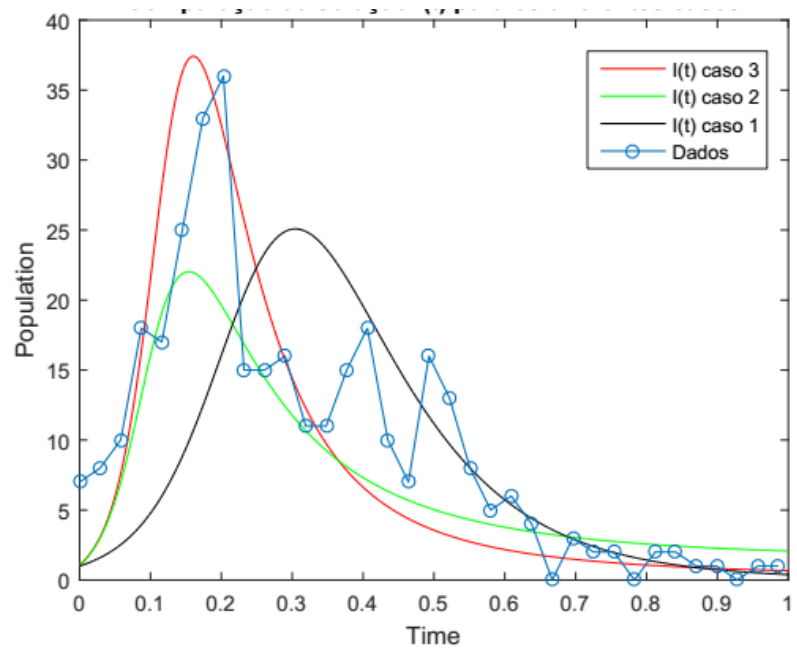

Figura 5: Comparação entre todos os casos.

Percebemos que o último caso apresentado é o que melhor reflete os dados reais, assim na Figura 5 , conseguimos visualizar para cada um dos casos o comportamento da curva $I(t)$ com os dados reais. Nessa figura, a curva $(I(t)$ caso 3$)$ representa $\theta_{1}=0,99, \theta_{2}=0,8, \theta_{3}=0,6, \theta_{4}=0,7$, como $(I(t)$ caso 2$)$, temos $\theta_{1}=\theta_{2}=\theta_{3}=\theta_{4}=0,8$ e a curva denominada $(I(t)$ caso 1$)$ temos $\theta_{1}=$ $\theta_{2}=\theta_{3}=\theta_{4}=1$. 


\section{CONCLUSÃo}

Neste trabalho nosso interesse está em ressaltar a importância de se analisar com modelos epidemiológicos, principalmente buscando novas abordagens que fazem com que os modelos se aproximem cada vez mais da realidade.

Apresentamos o PVI fracionário Eq.(1), trabalhado com múltiplas ordens de derivada, garantindo sua boa colocação, já que sem esse resultado as simulações não são válidas.

Além disso, apresentamos uma comparação numérica, buscando mostrar as diferenças no comportamento do modelo quando utilizamos diferentes ordens para a derivada, obtendo a curva com maior semelhança aos dados reais.

Conseguimos garantir que existe uma aproximação aos dados reais quando utilizamos derivadas de ordem fracionária segundo Caputo. Além do mais, mostramos que a escolha de estudar o PVI fracionário Eq.(1) $\operatorname{com} \theta_{j}$ iguais, implicando que a memória epidemiológica está sendo considerada a mesma em todas linhas do sistema não é a modelagem mais realista. Visto que quando consideramos ordens diferentes, a aproximação da curva $I(t)$ com os dados reais fica melhor.

Apesar de encontrarmos uma boa combinação de ordens fracionárias para essa dinâmica, não conseguimos garantir que esta é a melhor combinação possível, sendo essa questão objetivo futuro de nossos estudos.

\section{AGRADECIMENTOS}

Agradecemos ao Programa de Pós-Graduação em Modelagem Computacional, a Universidade Federal do Rio Grande e a Coordenação de Aperfeiçoamento de Pessoal de Nível Superior.

\section{REFERÊNCIAS BIBLIOGRÁFICAS}

1. Casagrandi R, Bolzoni L, Levin SA, Andreasen V. The SIRC model and influenza A. Math biosci. 2006 Apr;200(2):152-169, doi: 10.1016/j.mbs.2005.12.029.

2. Last JM, et al. A dictionary of epidemiology. International Epidemiological Association, Inc., 2001.

3. Yang HM. Epidemiologia matemática: Estudos dos efeitos da vacinação em doenças de transmissão direta. Editora da UNICAMP, 2001.

4. DeWitte SN, Wood JW. Selectivity of Black Death mortality with respect to preexisting health. Proc Natl Acad Sci. 2008 Jun;105(5):1436-1441, doi: 10.1073/pnas.0705460105.

5. Santos LADC. Um século de cólera: itinerário do medo. Physis: Rev Saúde Colet 1994 4(1):79-110.

6. Almeida AC, et al. Multiple source spatial cluster detection via multi-criteria analysis. Environ Ecol Stat. 2018 Jun;25(2):1-19, doi: 10.1007/s10651-018-0403-9.

7. Hays JN. Epidemics and pandemics: their impacts on human history. Abc-clio, 2005.

8. Luiz MH. Modelos Matemáticos em Epidemiologia [dissertação]. São Paulo (SP): Universidade Estadual Paulista; 2012.

9. Kermack WO., McKendrick, AG. A contribution to the mathematical theory of epidemics. Proc R Soc Lond. Series A. 1927 Ago;115(772):700-721, doi:10.1098/rspa.1927.0118.

10. Cruvinel WM, et al. Sistema imunitário: Parte I. Fundamentos da imunidade inata com ênfase nos mecanismos moleculares e celulares da resposta inflamatória. Rev Bras Reumat. 2010;50(4):434-61.

11. Medzhitov R, Janeway CA. Innate immunity: the virtues of a nonclonal system of recognition. Cell. 1997 Out;91(3):295-298, doi: 0.1016/S0092-8674(00)80412-2.

12. Mesquita Júnior D, et al. Sistema imunitário-parte II: fundamentos da resposta imunológica mediada por linfócitos T e B. Rev Bras Reumat. 2010;50(5):552-80.

13. Diethelm, K. The analysis of fractional differential equations: An application-oriented exposition using differential operators of Caputo type. Springer Science \& Business Media, 2010.

14. Pimenov A, et al. Memory effects in population dynamics: spread of infectious disease as a case study. Math Model Nat Phenom. 2012 Jun;7(3):204-226, doi: 10.1051/mmnp/20127313

15. Khader MM, Babatin, MM. Legendre spectral collocation method for solving fractional SIRC model and influenza A. J Comput Anal Appl. 2014 Out;17(2): 214-229.

16. El-Shahed M, Alsaedi A. The fractional SIRC model and influenza A. Math Problems Eng. 2011 Ago;2011:1-9, doi:10.1155/2011/480378.

17. Rihan FA, et al. On fractional SIRC model with salmonella bacterial infection. Abstr Appl Anal. 2014 Abr:2014:1-9, doi: 10.1155/2014/136263. 
18. Gomes ACFN, De Cezaro A. Um estudo sobre a memória epidemiológica: modelo SIRC fracionário. Rev Eletr Paulista Matem. 2017 Dez;10:194-210.

19. Paes NA, Silva LAA. Doenças infecciosas e parasitárias no Brasil: uma década de transição. Rev Panam Salud Pública, 1999;6(2):99-109.

20. Cabral CM, et al. Descrição clínico-epidemiológica dos nascidos vivos com microcefalia no estado de Sergipe, 2015. Epidemiol Serv Saúde. 2017 Jun;26(2):245-254, doi: 10.5123/S167949742017000200002.

21. Vargas A, et al. Características dos primeiros casos de microcefalia possivelmente relacionados ao vírus Zika notificados na Região Metropolitana de Recife, Pernambuco. Epidemiol Serv Saúde. 2016 Dez;25(4):691-700, doi: 10.5123/S1679-49742016000400003.

22. Malta JMAS, et al. Síndrome de Guillain-Barré e outras manifestações neurológicas possivelmente relacionadas à infecção pelo vírus Zika em municípios da Bahia, 2015. Epidemiol Serv Saúde. 2017 Mar:26(1):9-18, doi: 10.5123/S1679-49742017000100002.

23. Mota JCD, et al. Estimativa de taxa de mortalidade e taxa de incidência de sequelas cardíacas e digestivas por doença de Chagas no Brasil, 2008. Epidemiol Serv Saúde. 2014 Dez;23(4):711-720, doi: 10.5123/S1679-49742014000400013.

24. Böhm AW, et al. Tendência da incidência de dengue no Brasil, 2002-2012. Epidemiol Serv Saúde. 2016 Dez;25(4):725-733, doi:1 0.5123/S1679-49742016000400006.

25. Saad LDC, Barata RB. Surtos de febre amarela no estado de São Paulo, 2000-2010. Epidemiol Serv Saúde. 2016 Set;25(3):531-540. 